\title{
Expression profile of circular RNAs in the peripheral blood of neonates with hypoxic-ischemic encephalopathy
}

\author{
XIAOHUA DONG $^{1 *}$, SISI ZHUANG ${ }^{2,3^{*}}$, YUN HUANG $^{1}$, XIAOJING YANG $^{1}$, \\ YANRONG FU ${ }^{4}$, LINGLING $\mathrm{YU}^{1}$ and YINGMIN ZHAO ${ }^{1}$ \\ ${ }^{1}$ Department of Pediatric, Jingjiang People's Hospital Affiliated to Yangzhou University, Jingjiang, Jiangsu 214500; \\ ${ }^{2}$ Department of Pediatrics, The First Affiliated Hospital, Nanjing Medical University, Nanjing, Jiangsu 210036; \\ ${ }^{3}$ Nanjing Maternal and Child Health Medical Institute, Women's Hospital of Nanjing Medical University \\ (Nanjing Maternity and Child Health Care Hospital), Nanjing, Jiangsu 210004; ${ }^{4}$ Tongren Hospital, \\ Shanghai Jiao Tong University School of Medicine, Shanghai 200336, P.R. China
}

Received June 12, 2019; Accepted February 24, 2020

DOI: $10.3892 / \mathrm{mmr} .2020 .11091$

\begin{abstract}
Circular RNAs (circRNAs) are a class of non-coding RNAs that participate in various biological processes. However, the function of circRNAs in neonatal hypoxic-ischemic encephalopathy (HIE) is not fully understood. In the present study, the differentially expressed circRNAs in the peripheral blood of neonates with HIE and control samples were characterized by a microarray assay. A total of 456 circRNAs were significantly differentially expressed in the peripheral blood of neonates with HIE, with 250 upregulated and 206 downregulated circRNAs in HIE compared with the control samples. Reverse transcription-quantitative PCR was used to investigate specific circRNAs. Gene Ontology, and Kyoto Encyclopedia of Genes and Genomes pathway analyses were used to determine the function of the parent genes of the dysregulated circRNAs. In addition, microRNAs that may be associated with specific circRNAs were predicted using miRanda. Collectively, the present results indicated the potential importance of circRNAs in the peripheral blood of neonates with HIE.
\end{abstract}

\section{Introduction}

Neonatal hypoxic-ischemic encephalopathy (HIE) is mainly triggered by perinatal asphyxia, leading to neural tissue damage caused by deprivation of oxygen and glucose $(1,2)$. The

Correspondence to: Miss Yingmin Zhao or Miss Lingling Yu, Department of Pediatric, Jingjiang People's Hospital Affiliated to Yangzhou University, 28 Zhongzhou Road, Jingjiang, Jiangsu 214500, P.R. China

E-mail: yingmin_hosp@126.com

E-mail: xiaofiahyu@163.com

${ }^{*}$ Contributed equally

Key words: hypoxic-ischemic encephalopathy, circular RNA, microRNA, microarray pathophysiology of HIE is complex and may be related to complications in the neonate, mother or placenta (3). Cerebral hypoxic ischemia causes a strong neuroinflammatory response, which results in delayed cell death. Neonatal HIE can cause mortality or major disability, as well as cerebral palsy, neuromotor and cognitive delays, growth restriction and epilepsy (4-8).

Hypothermic therapy is an effective treatment for moderate and severe HIE by improving the neurological function of survivors (8). Effective hypothermia therapy can enhance neural repair, which is thought to improve neurological outcomes (9). However, current examinations of neonatal HIE have limitations and different levels of effectiveness, as diagnosis largely depends on clinical judgement and instrumental examination (10). Therefore, effective methods to define the degree of insult of HIE are required urgently.

Circular RNAs (circRNAs) are a class of non-coding RNAs that are produced from precursor mRNA back-splicing of exons (11). The downstream 5' splice site and upstream 3' splice site of circRNA are ligated by a $3^{\prime}-5$ ' phosphodiester bond at the junction site $(11,12)$. Previous studies have shown that circRNAs play essential roles in neuronal function, cell proliferation and immune responses (13-15). Furthermore, circRNAs are usually stable both in extracellular and intracellular environments $(16,17)$. However, the expression profiles and functions of circRNAs in neonatal HIE have not been previously reported.

In the present study, the expression profile of circRNAs in three neonatal HIE samples and control samples were detected by microarray analysis. Additionally, Gene Ontology (GO), and Kyoto Encyclopedia of Genes and Genomes (KEGG) pathway analyses results suggested that several processes were enriched in the HIE group compared with the control group, and are related to the cellular processes, immune system, signal transduction and cellular community. Thus, the present results may provide a novel insight into the progress of neonatal HIE.

\section{Materials and methods}

Sample collection. Samples were collected from Jingjiang People's Hospital from Aug, 2017 to May, 2018. Peripheral 
blood samples $(6 \mathrm{ml})$ from ten neonates with HIE and ten control infants without HIE were collected. The clinical parameters of neonatal patients and controls are listed in Table I. A diagnosis of HIE was confirmed by routine examination; An Apgar score of 2-6 for $1 \mathrm{~min}$ and $<8$ after first 5 min after birth indicates HIE (18) (Table I). PAXgene Blood RNA tubes (Qiagen $\mathrm{GmbH}$ ) with $4 \mathrm{ml}$ RNA protect reagent were used for collection of peripheral blood samples. All HIE samples were collected prior to hypothermia therapy. The present study was approved by the Medical Ethics Committee of Jingjiang People's Hospital in China [approval no. (2017)25].

Total RNA extraction and purification. Total RNA was extracted and purified from the whole blood sample using the PAXgene Blood RNA kit (Qiagen, Inc.) following the manufacturer's instructions. Total RNA was then checked for RNA integration using an Agilent Bioanalyzer 2100 (Agilent Technologies, Inc.).

Microarray analysis. Microarray was performed using an Agilent microarray platform (Shanghai Biotechnology Co., Ltd.), according to the manufacturer's protocols. Total RNA was amplified and labeled using a Low Input Quick Amp Labeling kit, One-Color (Agilent Technologies, Inc.), following the manufacturer's instructions. Then, the labeled cRNA were purified using a RNeasy mini kit (Qiagen, Inc.). Each slide was hybridized with $1.65 \mu \mathrm{g}$ Cy3-labeled cRNA using a Gene Expression Hybridization kit (Agilent Technologies, Inc.) in a hybridization oven at $65^{\circ} \mathrm{C}$ for $17 \mathrm{~h}$ (Agilent Technologies, Inc.), according to the manufacturer's protocol. After $17 \mathrm{~h}$ of hybridization, the slides were washed in staining dishes (Thermo Shandon; Thermo Fisher Scientific, Inc.) with Gene Expression Wash Buffer kit (Agilent Technologies, Inc.), following the manufacturer's instructions. Then, slides were scanned by an Agilent Microarray scanner (Agilent Technologies, Inc.) with the following default settings: Dye channel, green; Scan resolution $=3 \mu \mathrm{m}$; photomultiplier tube 100\%, 20 bit. Data were extracted using Feature Extraction software 10.7 (Agilent Technologies, Inc.). Raw data were normalized using a Quantile algorithm, according to the manufacturer's instructions provided by the microarray.

RNA-sequence analysis of $H I F-1 \alpha$. The microarray data discussed in the present study, with regards to the expression of $H I F-l \alpha$, have been deposited in the National Center for Biotechnology Information Gene Expression Omnibus (GEO) database. The GEO accession number is GSE121178.

GO and KEGG pathway analysis. The GO and KEGG pathway was predicted using Amigo 2 (http://amigo.geneontology.org/; V2.5.12) and GenomeNet website (http://www. genome.ad.jp/kegg/).

Reverse transcription-quantitative PCR (RT-qPCR). Total RNA was extracted as described above. RT-qPCR was performed using HiScript II Q RT SuperMix for qPCR kit (Vazyme Biotech Co., Ltd.) and SYBR Green (Roche Diagnostics) method following the manufacturer's protocol. To investigate the results of the microarray analysis, the primers of
Table I. Population demographics.

\begin{tabular}{lcl}
\hline Characteristic & HIE $(\mathrm{n}=10)$ & Control $(\mathrm{n}=10)$ \\
\hline Age, day & $1(1-2)$ & $5(2-10)$ \\
Sex, M/F & $6 / 4$ & $4 / 6$ \\
1 min Apgar & $3(2-6)$ & $10(9-10)$ \\
5 min Apgar & $7(6-8)$ & $10(10-10)$ \\
Cord pH & $7.34(7.245-7.442)$ & - \\
\hline
\end{tabular}

Data in the brackets represent the minimum and maximum of the medical index. M, male; F, female; HIE, Hypoxic Ischemic Encephalopathy. HIE group included six moderate HIE and four severe HIE cases.

circRNA were designed by CircPrimer software (http://www. bioinf.com.cn; V1.2), and the sequences of primers used are listed in Table II. RT-qPCR was performed using an Applied Biosystems ViiA 7 Dx system (Thermo Fisher Scientific, Inc.) with SYBR Green (Roche Diagnostics). The qPCR conditions were as follows: Initial denaturation at $50^{\circ} \mathrm{C}$ for $2 \mathrm{~min}, 95^{\circ} \mathrm{C}$ for $10 \mathrm{~min}$, followed by 40 cycle at $95^{\circ} \mathrm{C}$ for $15 \mathrm{sec}$ and $60^{\circ} \mathrm{C}$ for $30 \mathrm{sec}$. The expression of circRNA was normalized to the $18 \mathrm{~S}$ ribosomal RNA, using the $2^{-\Delta \Delta \mathrm{Cq}}$ method (19).

circRNA and microRNA (miRNA) interaction prediction. The circRNA and microRNA interaction was predicted using miRanda (http://www.microrna.org/; V21.0). The network map of circRNA and microRNA was drawn using Cytoscape (https://cytoscape.org; V3.7.2).

Statistical analysis. Data were analyzed using the SPSS 20.0 software package (SPSS, Inc.) with an independent-sample t-test for comparisons between the HIE and control group. All experiments were repeated three times. Data are presented as the mean $\pm \mathrm{SD}$. $\mathrm{P}<0.05$ was considered to indicate a statistically significant difference.

\section{Results}

circRNA microarray profiling. Peripheral blood samples were collected from ten neonates with HIE and ten infants without HIE. The 10 HIE samples included samples from six infants with moderate HIE and four with severe HIE. Demographic data of the HIE group and non-HIE groups are shown in Table I. To detect the differentially expressed circRNAs in HIE, microarray profiling was performed on three randomly selected HIE samples and controls. A total of 88,750 circRNAs were detected, with 456 circRNAs found to be differentially expressed between the HIE samples and control samples. Hierarchical clustering showed the total number of differentially expressed circRNAs among the samples (Fig. 1A). A scatter plot was used to visualize the circRNAs with a fold change of $>2.0$. The red and green lines are the fold change lines for the differentially expressed circRNAs (Fig. 1B). Furthermore, a volcano plot was used to visualize the differentially expressed circRNAs with fold changes, $>2.0$ and with statistical significance $(\mathrm{P}<0.05$; 
Table II. Primer sequences used for reverse transcription-quantitative PCR of circular RNA expression.

\begin{tabular}{lll}
\hline CircID & \multicolumn{1}{c}{ Forward primer $\left(5^{\prime} \rightarrow 3^{\prime}\right)$} & Reverse primer $\left(5^{\prime} \rightarrow 3^{\prime}\right)$ \\
\hline has_circ_0005537 & GGAGAAGACCAGGCAGAAGA & TGGTCATGATTCATCCCAGCT \\
hsa_circ_0050345 & CCTGAGACCAAACTTACAGCC & ACGTGGCAAGGTAGACAGAT \\
hsa_circ_0050705 & GCCACCACTTTGAGACACTG & TGTAGTCCATCCGAACCCTG \\
hsa_circ_0069578 & TGGCTACTTTGGTTTCTGTCTG & CATCATGGGCTGCCTGTATG \\
hsa_circ_0070733 & TGTGATGATGGCTGGACTGA & CCACTGTGCCTTCAAACTCA
\end{tabular}

CircID, circular RNAs identity.

A

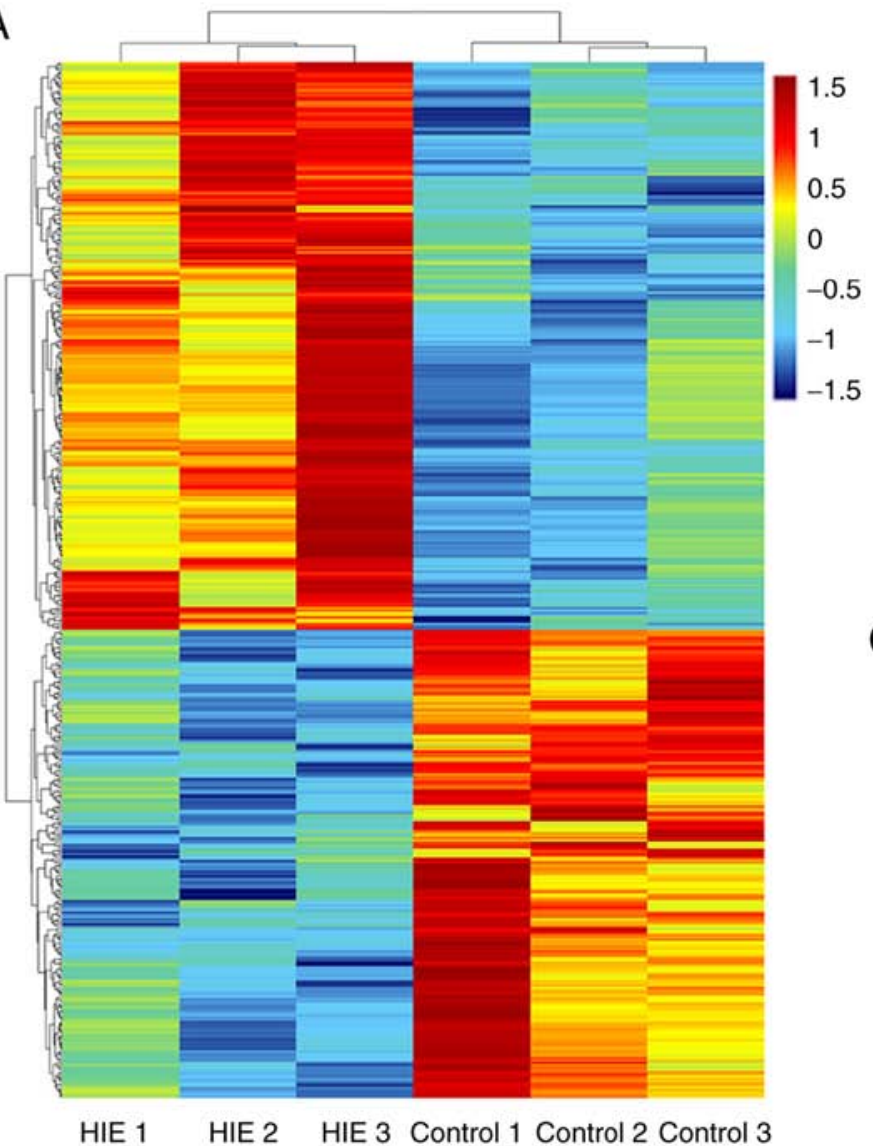

B

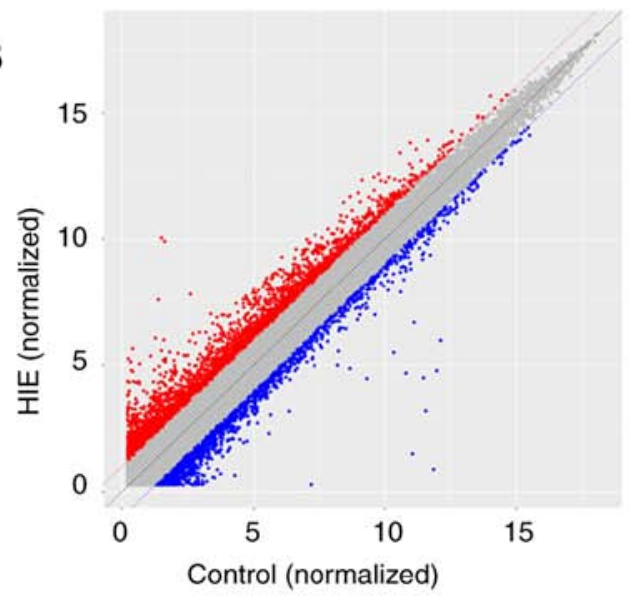

C

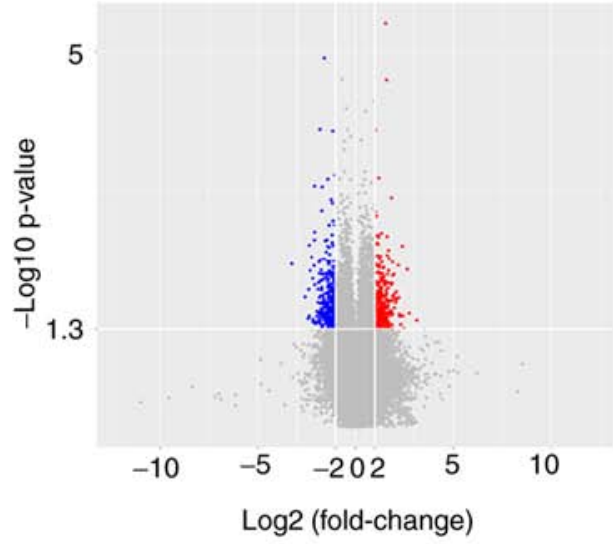

Figure 1. Analysis of differentially expressed circRNAs in HIE and control peripheral blood samples. (A) Heat map of differentially expressed circRNAs in peripheral blood from the HIE group compared with peripheral blood from the controls. (B) Scatter plots used to detect circRNA expression variation between the HIE and control groups. The circRNAs above the top red line and below the bottom green line indicated a $>2.0$-fold change of circRNAs between the HIE and control samples. (C) Volcano plots of differentially expressed circRNAs between the two samples. The blue and red points in the plot represents the downregulated and upregulated expressed circRNAs with statistical significance, respectively. circRNA, circular RNA; HIE, hypoxic-ischemic encephalopathy.

Fig. 1C). The present results suggested that 456 circRNAs, including 250 upregulated and 206 downregulated circRNAs, were differentially expressed in the HIE samples compared with the non-HIE samples. Tables III and IV demonstrated the characteristics of the top 20 upregulated circRNAs and the top 20 downregulated circRNAs, respectively (fold change, $\geq 2 ; \mathrm{P}<0.05)$.

$R T-q P C R$ validation. To further investigate the microarray analysis results, the present study randomly selected five differentially expressed circRNAs in the Tables III and IV, including three upregulated circRNAs (circRNA_0005537; circRNA_0050345; circRNA_0050705) and two downregulated circRNAs (circRNA_0069578; circRNA_0070733). RT-qPCR analysis was performed on additional HIE samples and non-HIE samples. The present results suggested that the mRNA expression levels of the selected circRNAs were significantly different in the HIE samples compared with the control samples (Fig. 2). Compared with the control samples, the expression levels of circRNA_0005537, circRNA_0050705 and circRNA_0050345 in the HIE samples was 5.21-, 2.32and 2.59-fold higher, respectively. By contrast, the expression 
Table III. Top 20 upregulated circRNAs in the hypoxic ischemic encephalopathy peripheral blood samples compared with normal blood samples.

\begin{tabular}{lllll}
\hline circRNA & P-value & FC & circRNA_length (bp) & Gene symbol \\
\hline hsa_circ_0015493 & 0.007985563 & 6.32061115 & 341 & QSOXI \\
hsa_circ_0084605 & 0.003891836 & 5.277928402 & 1661 & ASPH \\
hsa_circ_0080208 & 0.032305489 & 4.829873143 & 6577 & GRB10 \\
hsa_circ_0017745 & 0.031672451 & 4.771273975 & 313 & DHTKD1 \\
hsa_circ_0085851 & 0.019112436 & 4.676079992 & 1269 & TSTA3 \\
hsa_circ_0050345 & 0.007019217 & 4.596592582 & 617 & DPY19L3 \\
hsa_circ_0005537 & 0.019407829 & 4.111283582 & 326 & CDC25C \\
hsa_circ0034212 & 0.009278198 & 3.938135751 & 2362 & HERC2 \\
hsa_circ_0014223 & 0.009610085 & 3.769487918 & 523 & S100A8 \\
hsa_circ_0088696 & 0.006352905 & 3.743285501 & 718 & STXBP1 \\
hsa_circ_0050705 & 0.049517311 & 3.618324961 & 1452 & WDR62 \\
hsa_circ_0043217 & 0.032711809 & 3.572406618 & 4544 & ACACA \\
hsa_circ_0080599 & 0.02837951 & 3.530978904 & 522 & GTF2IRD1 \\
hsa_circ_0083857 & 0.012124746 & 3.307009337 & 708 & GSR \\
hsa_circ_0033633 & 0.040119053 & 3.253147202 & 255 & C14Orf80 \\
hsa_circ_0064377 & 0.014170302 & 3.212198165 & 3786 & CAND2 \\
hsa_circ_0045827 & 0.043441186 & 3.178805121 & 1976 & MXRA7 \\
hsa_circ_0085853 & 0.024352592 & 3.178538435 & 65 & TSTA3 \\
hsa_circ_0010211 & 0.025240307 & 3.165611241 & 1788 & ARHGEF1OL \\
hsa_circ_0067605 & 0.009250664 & 3.16140903 & 378 & GK5 \\
\hline
\end{tabular}

circRNA, circular RNA; FC, Fold change.

Table IV. Top 20 downregulated circRNAs in the hypoxic ischemic encephalopathy peripheral blood samples compared with normal blood samples.

\begin{tabular}{lccrl}
\hline circRNA & P-value & FC & circRNA_length (bp) & Gene symbol \\
\hline hsa_circ_0051858 & 0.006718587 & 9.691469839 & 1856 & TRPM4 \\
hsa_circ_0077755 & 0.018710732 & 6.059898228 & 2896 & GJAl \\
hsa_circ_0012164 & 0.036270185 & 5.413530234 & 662 & KIF2C \\
hsa_circ_0061590 & 0.014796495 & 5.306206578 & 4364 & SETD4 \\
hsa_circ_0076770 & 0.008277684 & 5.021701979 & 2765 & LOC730101 \\
hsa_circ_0075624 & 0.010552438 & 4.49619749 & 271 & SYCP2L \\
hsa_circ0069578 & 0.040281981 & 4.492267813 & 617 & UCHL1 \\
hsa_circ_0035951 & 0.003210402 & 4.217652612 & 729 & DENND4A \\
hsa_circ0062272 & 0.002515151 & 4.197587878 & 2975 & SEPT5-GP1BB \\
hsa_circ_0070733 & 0.042574192 & 4.072228574 & 790 & PRSS12 \\
hsa_circ_0080184 & 0.039725995 & 3.946056497 & 11042 & TNS3 \\
hsa_circ_0056518 & 0.033688712 & 3.852150499 & 126 & NCKAP5 \\
hsa_circ_0073280 & 0.040374199 & 3.717489627 & 2346 & GPR98 \\
hsa_circ0009506 & 0.022064444 & 3.708353404 & 117 & ACOT7 \\
hsa_circ_0008091 & 0.009072897 & 3.543094344 & 405 & NDUFS1 \\
hsa_circ0025111 & 0.019091997 & 3.526641269 & 241 & VWF \\
hsa_circ_0034711 & 0.042187121 & 3.456252673 & 4006 & RPAP1 \\
hsa_circ0071519 & 0.038634183 & 3.451687321 & 2419 & STOX2 \\
hsa_circ_0077888 & 0.031798526 & 3.429293915 & 1469 & EYA4 \\
hsa_circ_0062751 & 0.005900349 & 3.389322219 & 2051 & GAS2L1 \\
\hline
\end{tabular}

circRNA, circular RNA; FC, Fold change. 


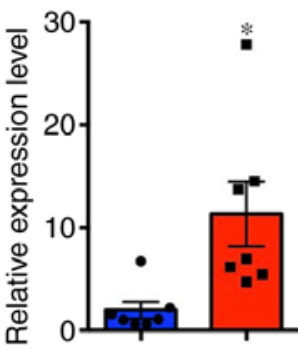

Circ_0005537

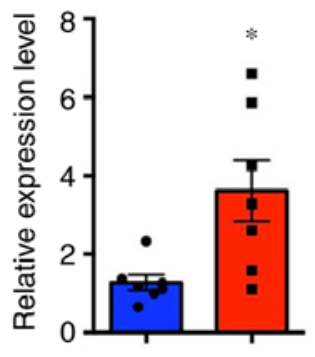

Circ_0050345

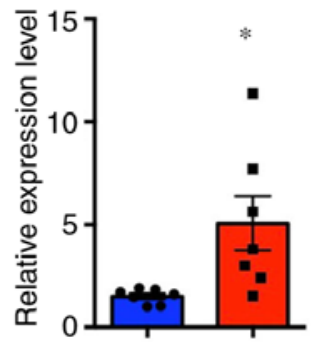

Circ_0050705

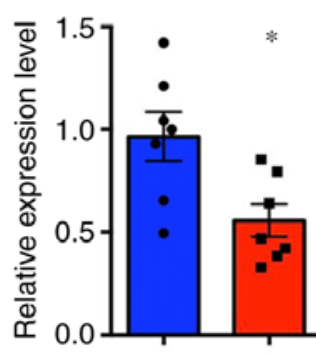

Circ_0069578

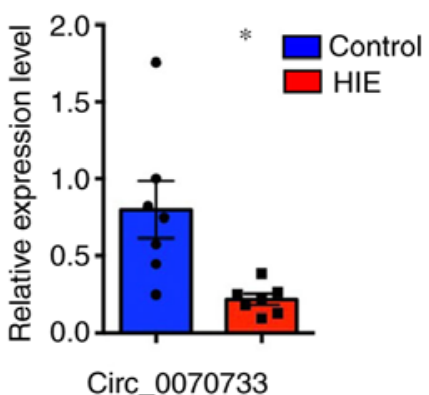

Figure 2. Reverse transcription-quantitative PCR validation. Differential expression of circRNAs in the blood samples between neonatal hypoxic-ischemic encephalopathy and controls. ${ }^{*} \mathrm{P}<0.05$. circRNA, circular RNA.

levels of circRNA_0070733 and circRNA_0069578 in the HIE samples was 3.58- and 1.84-fold lower, respectively, compared with the control samples.

GO and KEGG pathway analysis of host genes of the circRNAs in HIE. Previous studies have shown that circRNAs function by regulating the expression levels of their parent genes at the transcriptional level $(20,21)$. To further identify the function of host genes of the differentially expressed circRNAs in HIE, GO and KEGG pathway analysis of the host genes was performed (Figs. 3 and 4). The GO results demonstrated that the host genes of the differentially expressed circRNAs are primarily involved in cellular processes (22), cell, cell part and binding (Fig. 3A), specifically in regulation of phospholipase $\mathrm{C}$ activity, hindbrain morphogenesis and fatty-acyl-CoA biosynthetic process (Fig. 3B). KEGG analysis results indicated that the immune system, signal transduction and cellular community (Fig. 4A), and in particular in the regulation of actin cytoskeleton, focal adhesion and ECM-receptor interaction (Fig. 4B), were related to the host genes of the differentially expressed circRNAs.

Interactions between circRNAs and miRNAs. Previous studies have shown that circRNAs can bind to miRNAs via miRNA response elements (MREs) and function as miRNA sponges $(13,23)$. To investigate the potential miRNAs that may bind to circRNAs, miRanda was used to screen the MREs in the five validated circRNAs. The present results indicated that several miRNAs were associated with specific circRNAs (Table V). A total of 92 miRNAs were identified to potentially bind to circRNA_0050705. It was also found that 16 miRNAs could potentially bind to circRNA_0005537, nine miRNAs could potentially bind to circRNA_0069578, eight miRNAs could potentially bind to circRNA_0070733 and six miRNAs could potentially bind to circRNA_0050345 (Fig. 5).

\section{Discussion}

Neonatal HIE is one of the common causes of death or permanent disability, particularly in developing countries $(24,25)$. The crucial role of miRNAs (miRs) and long non-coding RNAs in HIE pathologic processes have been previously demonstrated $(19,26)$. The expression level of miR-374a is significantly downregulated in the umbilical cord blood of neonatal HIE (26). Furthermore, miR-204 is reported to participate in the pathogenesis of HIE via the regulation of $K L L N(27)$.

circRNAs are a novel type of non-coding RNA that stably exist in peripheral blood (28) and play a crucial role in the regulation of numerous pathological or biological processes $(29,30)$. The expression profiles of circular RNAs in neonatal rats following hypoxic-ischemic brain damage (HIBD) has been previously reported (31). Jiang et al (31) identified that 66 circRNAs are differentially expressed in the early stages of HIBD. However, to the best of our knowledge, circRNA profiles determined by microarray analysis in the neonatal HIE have not been previously reported. Therefore, the present study investigated differentially expressed circRNAs in the peripheral blood of neonates with HIE and healthy controls using microarray analysis. The microarray expression profiles suggested that 250 upregulated circRNAs and 206 downregulated circRNAs were significantly differentially expressed in patients with HIE. Therefore, the distinct expression profile of circRNAs may participate in the pathogenesis of HIE and provide new biomarkers for HIE diagnosis. To further investigate the microarray data and identify potential clinically applicable biomarkers, the present study assessed five significant differentially expressed circRNAs in neonatal HIE. It was found that circRNA_0005537, circRNA_0050345 and circRNA_0050705 were significantly upregulated in patients with HIE compared with healthy controls, while circRNA_0069578 and circRNA_0070733 were downregulated.

Hypoxia-inducible factor $1 \alpha(\mathrm{HIF}-1 \alpha)$ is a sensitive molecule regulated by oxygen tension and mediates the adaptive response of the cell to a hypoxic environment (32). Zhu et al (33) reported that HIF-1 $\alpha$ can regulate BCL2 Interacting Protein 3 (BNIP3) by binding to the BNIP3 promoter in hypoxia-induced neurons, and participates in the process of HIE. The present results suggested that the expression level of $H I F-1 \alpha$ was upregulated in HIE group by RNA-seq (fold change, $>2$; data submitted to GEO; accession no. GSE121178). Previous studies have demonstrated that circRNAs may bind to miRNAs and regulate mRNAs expression (34). The present results indicated that miR-433 may potentially bind to circ_0005537, and that miR-338-3p could potentially bind to circ_0070733. However, microRNA.org was used to predict the miRNAs that could potentially bind to $H I F-l \alpha$, and found that HIF-1 $\alpha$ has miR-433 and miR-338-3p specific binding 
A

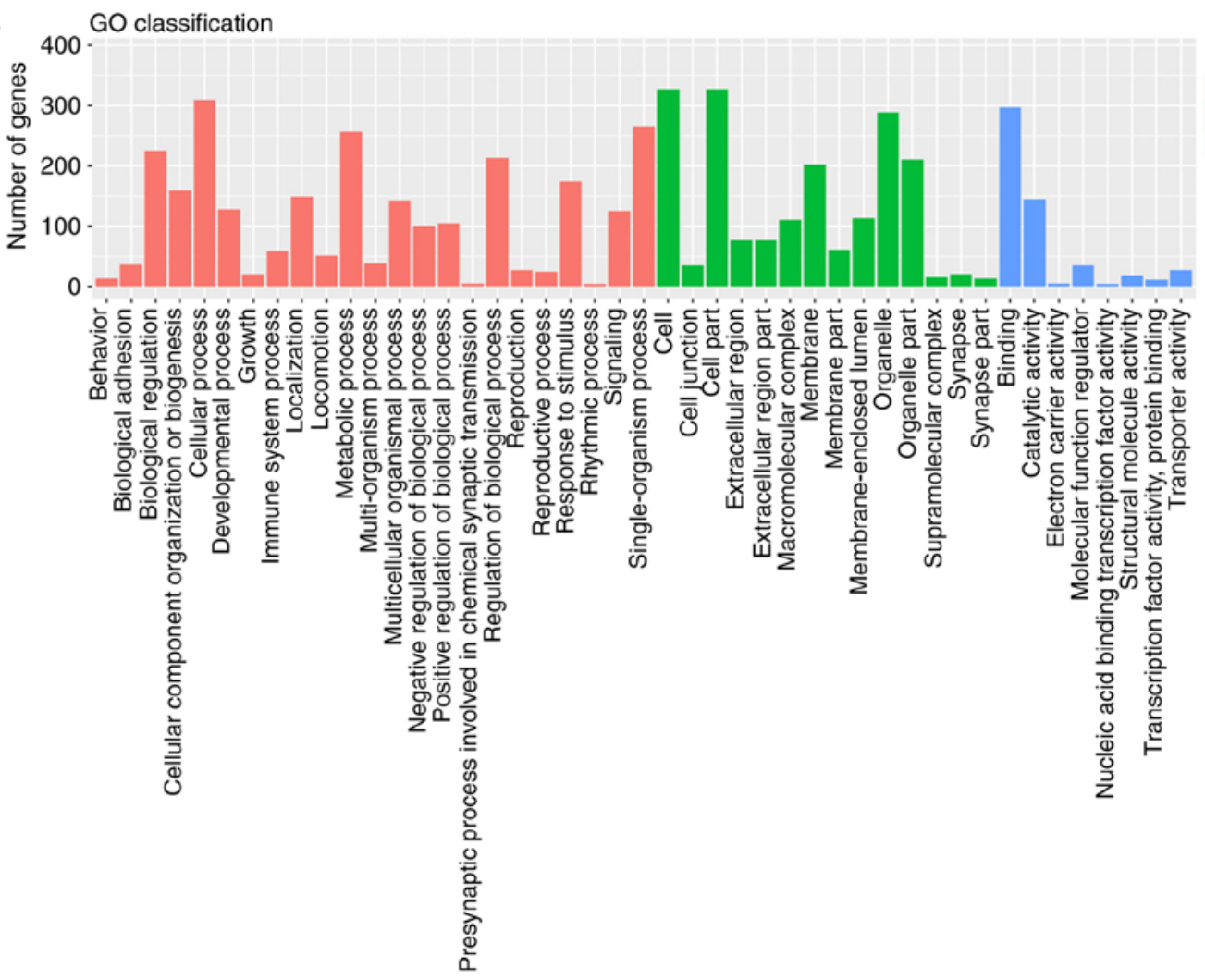

GO_domain

Biological_process

Cellular_componet

Molecular_gunction

B

Top 30 of GO enrichment

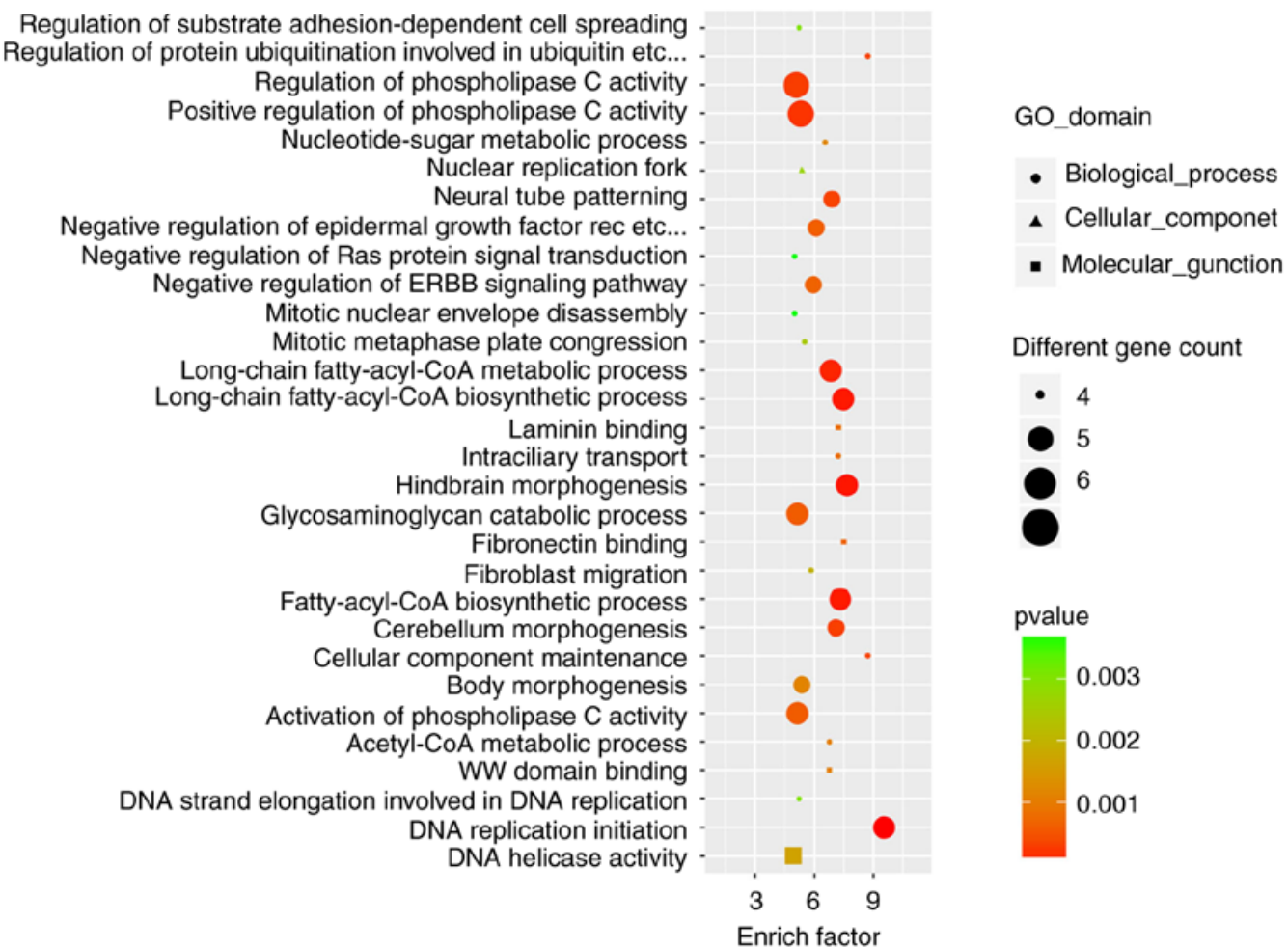

Figure 3. GO terms for host genes of dysregulated circRNAs between neonatal hypoxic-ischemic encephalopathy and control blood samples. (A) GO classification of differentially expressed circRNAs. (B) Top 30 circRNAs of GO enrichment. GO, Gene Ontology; circRNA, circular RNA.

sites (data not shown). Therefore, the present results suggested that dysregulated circ_0005537 and circ_0070733 in patients with HIE may regulate $H I F-1 \alpha$ expression, and participate in the pathological process of HIE.
circRNA lack crucial elements for cap-dependent translation, however, few endogenous circRNAs have been shown to act as protein templated $(35,36)$. According to the open reading frame prediction and functional internal ribosome 


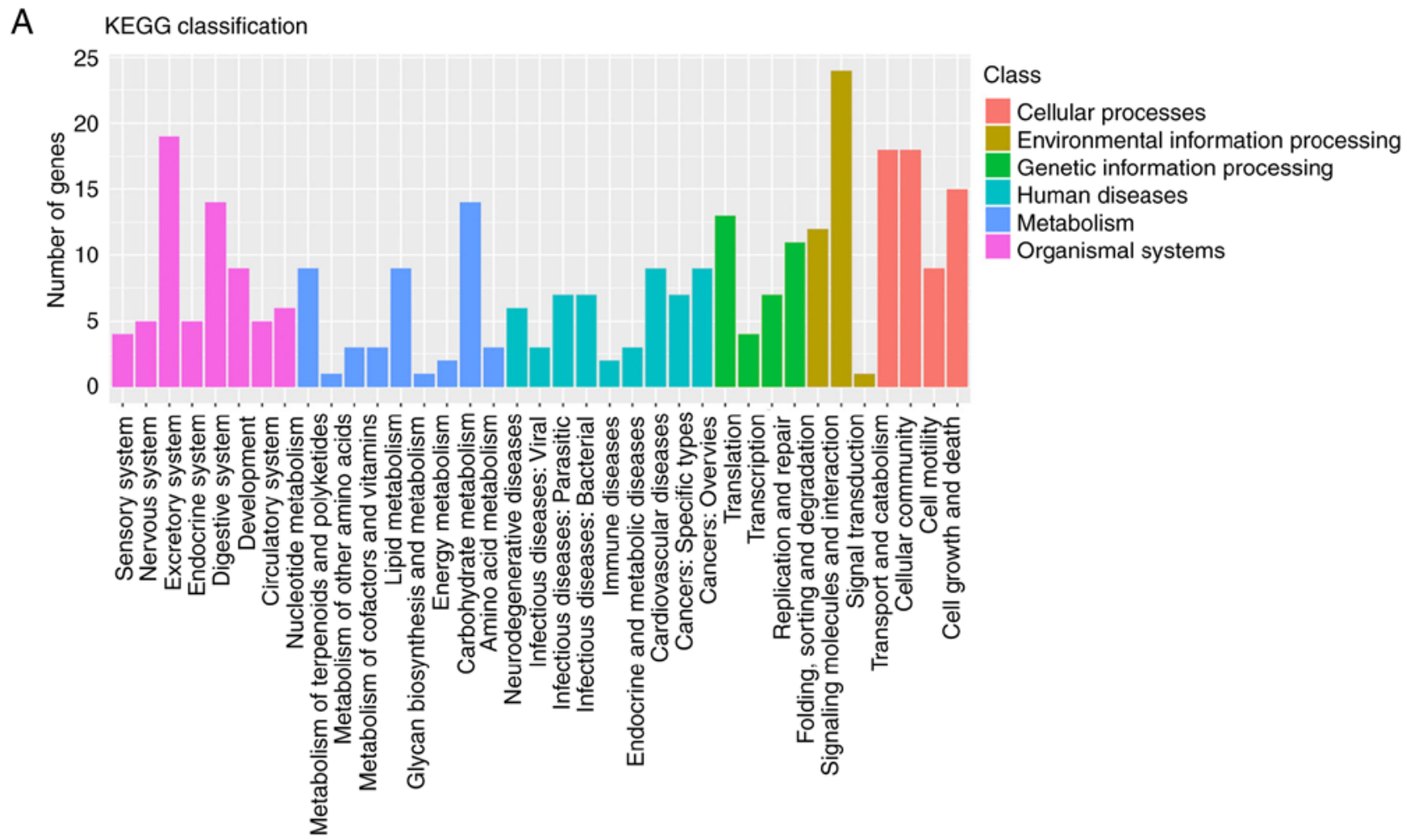

B

Top 30 of pathway enrichment

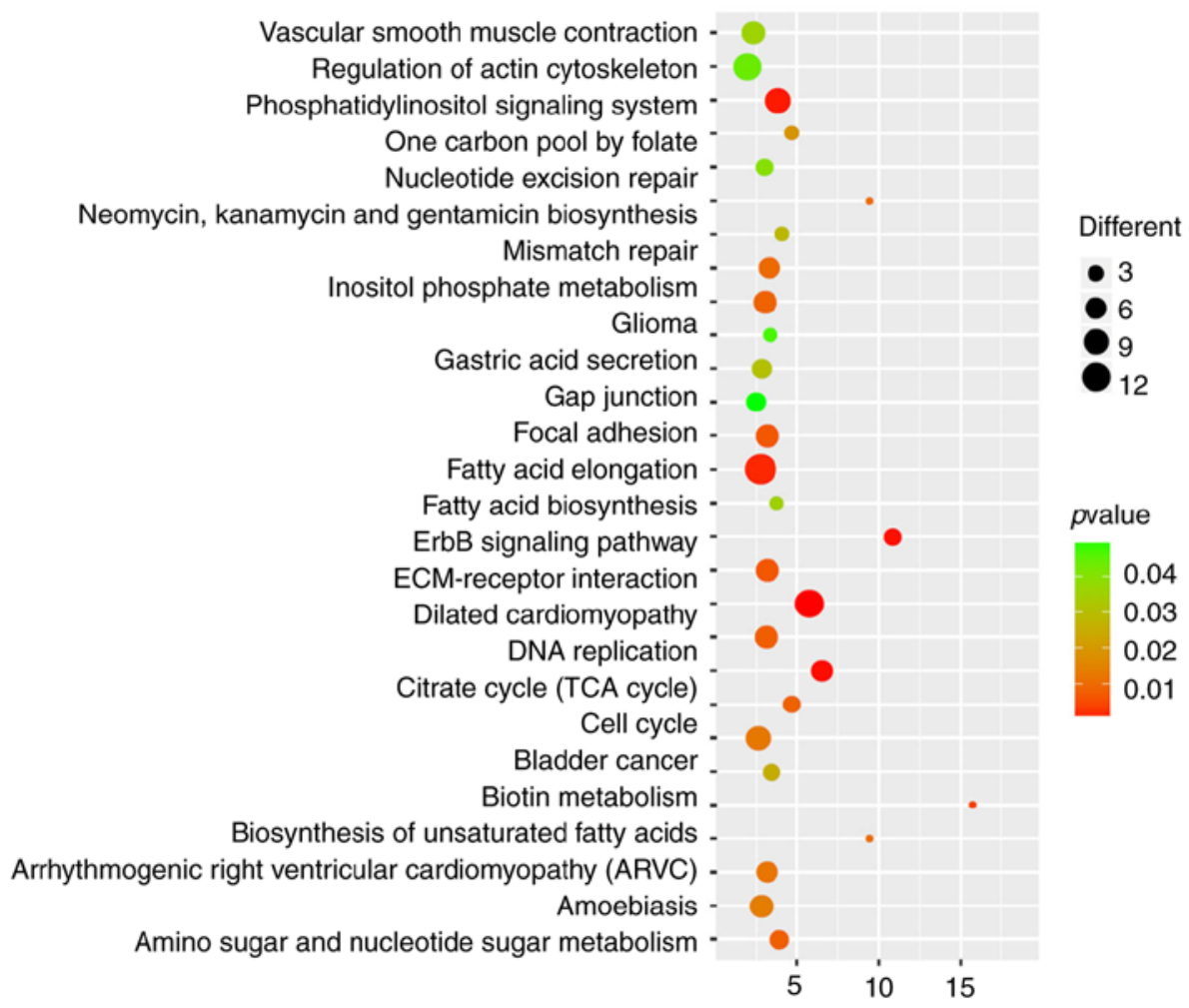

Figure 4. KEGG pathway analysis for host genes of dysregulated circRNAs between neonatal hypoxic-ischemic encephalopathy and control blood samples. (A) KEGG classification of differentially expressed circRNAs. (B) Top 30 circRNAs of pathway enrichment. KEGG, Kyoto Encyclopedia of Genes and Genomes; circRNA, circular RNA.

entry site element prediction of the circRNA_0005537, circRNA_0050345, circRNA_0050705, circRNA_0069578 and circRNA_0070733; it was suggested that these five circRNAs have the possibility to translate into short proteins or peptides (data not shown). However, the functional relevance of these five circRNA requires further study.

Brain ischemia leads to a lack of ATP and initiates a cascade of biochemical events, including cell death and the induction 
Table V. Interaction of circRNA and miRNAs.

circRNA

miRNA

miRNA number

circ_0005537 miR-1200, miR-1208, miR-1236, miR-1283, miR-1288, miR-1290, miR-136, miR-433,

miR-526b, miR-579, miR-587, miR-221-3p, miR-222-3p, miR-6131,miR-3199,

miR-8052

circ_0050345 miR-4717-5p, miR-518a-5p, miR-527, miR-505-5p, miR-4680-3p, miR-7974

circ_0050705 miR-8089, miR-4700-5p, miR-6738-3p, miR-637, miR-608, miR-6743-5p, miR-3187-5p,

miR-612, miR-6721-5p, miR-486-3p, miR-4731-5p, miR-370-3p, miR-1207-5p,

miR-6819-5p, miR-4739, miR-4690-5p, miR-6878-3p, miR-6734-5p, miR-3692-5p,

miR-6774-5p, miR-4632-5p, miR-1199-3p, miR-1908-5p, miR-3162-5p, miR-6744-3p,

miR-5088-5p, miR-149-5p, miR-6835-5p, miR-6738-5p, miR-6876-5p,miR-8069,

miR-615-5p, miR-6773-3p, miR-6796-5p, miR-4640-5p, miR-3132, miR-6737-5p,

miR-3907, miR-6742-5p, miR-6763-5p, miR-6860, miR-4726-3p, miR-6823-5p,

miR-4778-3p, miR-6842-3p, miR-3192-5p, miR-661, miR-4667-5p,miR-4459,

miR-6726-5p, miR-4749-5p, miR-6127, miR-3194-3p, miR-146b-3p,miR-6805-5p,

miR-5189-5p, miR-4476, miR-760, miR-4498, miR-611, miR-3180-3p, miR-345-5p,

miR-6821-5p, miR-7155-5p, miR-1229-5p, miR-1301-3p, miR-6791-5p, miR-5787,

miR-6893-3p, miR-6848-3p, miR-4664-5p, miR-1587, miR-6816-5p, miR-4763-3p,

miR-6865-5p, miR-1972, miR-6851-5p, miR-423-5p, miR-3922-3p, miR-6879-5p,

miR-6775-5p, miR-1251-3p, miR-1914-3p, miR-484, miR-6756-5p, miR-3184-5p,

miR-3620-5p, miR-3147, miR-885-3p, miR-3925-3p, miR-766-3p, miR-6852-5p

circ_0069578 miR-3158-5p, miR-3617-3p, miR-922, miR-4291, miR-761, miR-4725-3p, miR-6734-5p, miR-6780b-5p, miR-6825-5p

circ_0070733 miR-2355-5p, miR-6882-3p, miR-338-3p, miR-4286, miR-6762-3p, miR-24-3p,

miR-6716-5p, miR-4252

circRNA, circular RNA; miRNA/miR, microRNA.

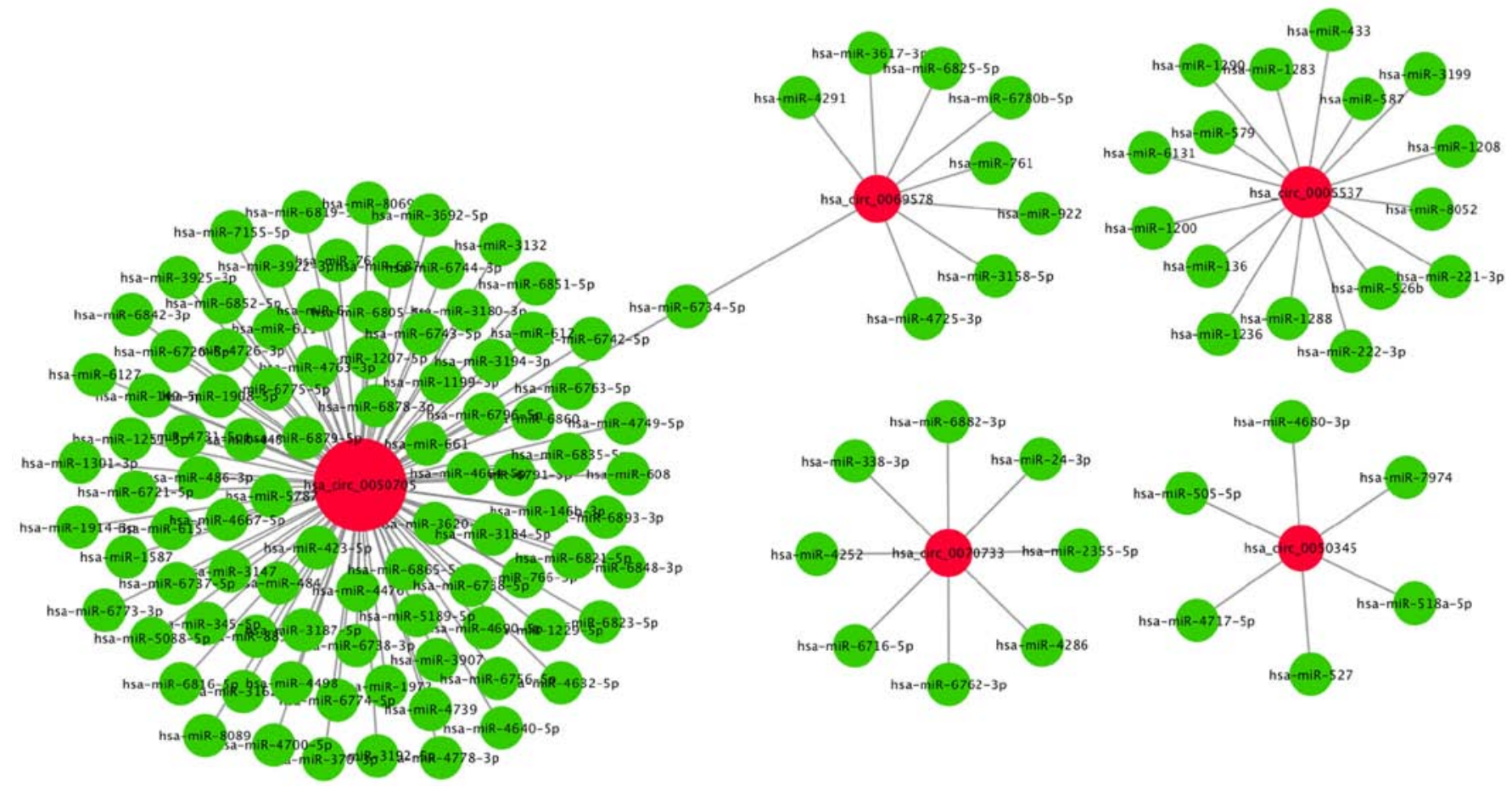

Figure 5. Network map of potential interaction between circRNAs and miRNAs. circ_0050705 had potential interactions with 92 miRNAs, circ_0005537 had potential interactions with 16 miRNAs, circ_0069578 had potential interactions with nine miRNAs, circ_0070733 had potential interactions with eight miRNAs and circ_0050345 had potential interactions with six miRNAs. circRNA, circular RNA; miRNA, microRNA. 
of secondary brain injury in neonates (37). In addition, glucose and oxygen play important roles in the pathogenesis of brain injury (1). Moreover, acetyl-CoA is essential in generating ATP from ADP in the mitochondrion $(1,38)$. In the present study, GO and KEGG pathway analyses results demonstrated that the host genes of dysregulated circRNAs participate in hindbrain morphogenesis and fatty-acyl-CoA biosynthetic processes. Therefore, the present results suggested that the dysregulated circRNAs may be involved in the pathogenesis of neonatal HIE.

In conclusion, microarray analysis was used to detect differentially expressed circRNAs between neonates with HIE and controls. To the best of our knowledge, the present study is the first to examine the potential relationship between circRNAs and HIE in neonates. In addition, the potential functions of the host genes of the dysregulated circRNAs were predicted by GO and KEGG pathway analyses. The GO and KEGG pathway analyses results indicated that the abnormally expressed circRNAs may be involved in the pathogenesis of HIE. However, whether the severity of HIE and the effect of hypothermia therapy are related to differentially expressed circRNAs in the progression of HIE requires further investigation.

\section{Acknowledgements}

Not applicable.

\section{Funding}

The current study was supported by grants from the National Natural Science Foundation of China (grant no. 81801503) and the Natural Science Foundation of Jiangsu Province (grant no. BK20180286).

\section{Availability of data and materials}

The microarray data that included the expression profile of circular RNAs and the expression profile of long non-coding RNAs (19) have been deposited in the National Center for Biotechnology Information GEO database (accession no. GSE121178). The datasets used and/or analyzed during the current study are available from the corresponding author on reasonable request.

\section{Authors' contributions}

YZ designed the project and edited the manuscript. LY analyzed the data and wrote the manuscript. XD designed the experiment and performed the bioinformatics analysis. SZ performed the bioinformatics analysis. $\mathrm{YH}$ and $\mathrm{XY}$ performed sample collection and statistical analysis. YF performed the data analysis. All authors read and approved the final manuscript.

\section{Ethics approval and consent to participate}

This work was approved by the Medical Ethics Committee of Jingjiang People's Hospital in China [approval no. (2017)25]. Informed consent was obtained from the guardian of subjects for participation in this study.

\section{Patient consent for publication}

Informed consent was obtained from the guardian of subjects for participation in this study.

\section{Competing interests}

The authors declare that they have no competing interests.

\section{References}

1. Verklan MT: The chilling details: Hypoxic-ischemic encephalopathy. J Perinat Neonatal Nurs 23: 59-68; quiz 69-70, 2009.

2. Qin X, Cheng J, Zhong Y, Mahgoub OK, Akter F, Fan Y, Aldughaim M, Xie Q, Qin L, Gu L, et al: Mechanism and treatment related to oxidative stress in neonatal hypoxic-ischemic encephalopathy. Front Mol Neurosci 12: 88, 2019.

3. Rainaldi MA and Perlman JM: Pathophysiology of birth asphyxia. Clin Perinatol 43: 409-422, 2016.

4. Shah PS, Ohlsson A and Perlman M: Hypothermia to treat neonatal hypoxic ischemic encephalopathy: Systematic review. Arch Pediatr Adolesc Med 161: 951-958, 2007.

5. Lai MC and Yang SN: Perinatal hypoxic-ischemic encephalopathy. J Biomed Biotechnol 2011: 609813, 2011.

6. Gluckman PD, Wyatt JS, Azzopardi D, Ballard R, Edwards AD, Ferriero DM, Polin RA, Robertson CM, Thoresen M, Whitelaw A and Gunn AJ: Selective head cooling with mild systemic hypothermia after neonatal encephalopathy: Multicentre randomised trial. Lancet 365: 663-670, 2005.

7. Shankaran S, Laptook AR, Ehrenkranz RA, Tyson JE, McDonald SA, Donovan EF, Fanaroff AA, Poole WK, Wright LL, Higgins RD, et al: Whole-body hypothermia for neonates with hypoxic-ischemic encephalopathy. N Engl J Med 353: 1574-1584, 2005.

8. Azzopardi DV, Strohm B, Edwards AD, Dyet L, Halliday HL, Juszczak E, Kapellou O, Levene M, Marlow N, Porter E, et al: Moderate hypothermia to treat perinatal asphyxial encephalopathy. N Engl J Med 361: 1349-1358, 2009.

9. Azzopardi D, Strohm B, Marlow N, Brocklehurst P, Deierl A, Eddama O, Goodwin J, Halliday HL, Juszczak E, Kapellou O, et al: Effects of hypothermia for perinatal asphyxia on childhood outcomes. N Engl J Med 371: 140-149, 2014.

10. Martinello K, Hart AR, Yap S, Mitra S and Robertson NJ: Management and investigation of neonatal encephalopathy: 2017 update. Arch Dis Child Fetal Neonatal Ed 102: F346-F358, 2017.

11. Chen LL: The biogenesis and emerging roles of circular RNAs. Nat Rev Mol Cell Biol 17: 205-211, 2016.

12. Wilusz JE: A $360^{\circ}$ view of circular RNAs: From biogenesis to functions. Wiley Interdiscip Rev RNA 9: e1478, 2018.

13. Li X, Yang L and Chen LL: The biogenesis, functions, and challenges of circular RNAs. Mol Cell 71: 428-442, 2018.

14. Yang L, Fu J and Zhou Y: Circular RNAs and their emerging roles in immune regulation. Front Immunol 9: 2977, 2018.

15. Sekar S and Liang WS: Circular RNA expression and function in the brain. Noncoding RNA Res 4: 23-29, 2019.

16. Li Y, Zheng Q, Bao C, Li S, Guo W, Zhao J, Chen D, Gu J, He X and Huang S: Circular RNA is enriched and stable in exosomes: A promising biomarker for cancer diagnosis. Cell Res 25: 981-984, 2015 .

17. Memczak S, Papavasileiou P, Peters $\mathrm{O}$ and Rajewsky N: Identification and characterization of circular RNAs as a new class of putative biomarkers in human blood. PLoS One 10: $\mathrm{e} 0141214,2015$

18. Thornberg E, Thiringer K, Odeback A and Milsom I: Birth asphyxia: Incidence, clinical course and outcome in a Swedish population. Acta Paediatr 84: 927-932, 1995.

19. Dong X, Zhao Y, Huang Y, Yu L, Yang X and Gao F: Analysis of long noncoding RNA expression profiles in the whole blood of neonates with hypoxic-ischemic encephalopathy. J Cell Biochem, Nov 26, 2018 (Epub ahead of print).

20. Li Z, Huang C, Bao C, Chen L, Lin M, Wang X, Zhong G, Yu B, $\mathrm{Hu}$ W, Dai L, et al: Exon-intron circular RNAs regulate transcription in the nucleus. Nat Struct Mol Biol 22: 256-264, 2015.

21. Zhang Y, Zhang XO, Chen T, Xiang JF, Yin QF, Xing YH, Zhu S, Yang L and Chen LL: Circular intronic long noncoding RNAs. Mol Cell 51: 792-806, 2013 
22. Ashburner M, Ball CA, Blake JA, Botstein D, Butler $\mathrm{H}$ Cherry JM, Davis AP, Dolinski K, Dwight SS, Eppig JT, et al: Gene ontology: Tool for the unification of biology. The gene ontology consortium. Nat Genet 25: 25-29, 2000.

23. Salmena L, Poliseno L, Tay Y, Kats L and Pandolfi PP: A ceRNA hypothesis: The rosetta stone of a hidden RNA language? Cell 146: 353-358, 2011.

24. Johnston MV, Fatemi A, Wilson MA and Northington F: Treatment advances in neonatal neuroprotection and neurointensive care. Lancet Neurol 10: 372-382, 2011.

25. Azra Haider B and Bhutta ZA: Birth asphyxia in developing countries: Current status and public health implications. Curr Probl Pediatr Adolesc Health Care 36: 178-188, 2006.

26. Looney AM, Walsh BH, Moloney G, Grenham S, Fagan A O'Keeffe GW, Clarke G, Cryan JF, Dinan TG, Boylan GB and Murray DM: Downregulation of umbilical cord blood levels of miR-374a in neonatal hypoxic ischemic encephalopathy. J Pediatr 167: 269-273.e2, 2015.

27. Chen R, Wang M, Fu S, Cao F, Duan P and Lu J: MicroRNA-204 may participate in the pathogenesis of hypoxic-ischemic encephalopathy through targeting KLLN. Exp Ther Med 18: 3299-3306, 2019.

28. Jakobi T and Dieterich C: Deep computational circular RNA analytics from RNA-seq data. Methods Mol Biol 1724: 9-25, 2018.

29. Memczak S, Jens M, Elefsinioti A, Torti F, Krueger J, Rybak A, Maier L, Mackowiak SD, Gregersen LH, Munschauer M, et al: Circular RNAs are a large class of animal RNAs with regulatory potency. Nature 495: 333-338, 2013.

30. Hansen TB, Jensen TI, Clausen BH, Bramsen JB, Finsen B, Damgaard CK and Kjems J: Natural RNA circles function as efficient microRNA sponges. Nature 495: 384-388, 2013.
31. Jiang L, Li H, Fan Z, Zhao R and Xia Z: Circular RNA expression profiles in neonatal rats following hypoxic-ischemic brain damage. Int J Mol Med 43: 1699-1708, 2019.

32. Wang GL, Jiang BH, Rue EA and Semenza GL: Hypoxia-inducible factor 1 is a basic-helix-loop-helix-PAS heterodimer regulated by cellular O2 tension. Proc Natl Acad Sci USA 92: 5510-5514, 1995.

33. Zhu L, Qi B and Hou D: Roles of HIF1 $\alpha$ - and HIF2 $\alpha$-regulated BNIP3 in hypoxia-induced injury of neurons. Pathol Res Pract 215: 822-827, 2019.

34. Li Y, Huo C, Lin X and Xu J: Computational identification of cross-talking ceRNAs. Adv Exp Med Biol 1094: 97-108, 2018.

35. Legnini I, Di Timoteo G, Rossi F, Morlando M, Briganti F, Sthandier O, Fatica A, Santini T, Andronache A, Wade M, et al: Circ-ZNF609 is a circular RNA that can be translated and functions in myogenesis. Mol Cell 66: 22-37.e9, 2017.

36. Pamudurti NR, Bartok O, Jens M, Ashwal-Fluss R, Stottmeister C, Ruhe L, Hanan M, Wyler E, Perez-Hernandez D, Ramberger E, et al: Translation of circRNAs. Mol Cell 66: 9-21.e7, 2017.

37. Thornton C, Leaw B, Mallard C, Nair S, Jinnai M and Hagberg H: Cell death in the developing brain after hypoxia-ischemia. Front Cell Neurosci 11: 248, 2017.

38. Vannucci RC, Brucklacher RM and Vannucci SJ: Glycolysis and perinatal hypoxic-ischemic brain damage. Dev Neurosci 27 $185-190,2005$

(i) $($ This work is licensed under a Creative Commons Attribution-NonCommercial-NoDerivatives 4.0 International (CC BY-NC-ND 4.0) License. 